\title{
Preferential transcription of Paracoccidioides brasiliensis genes: host niche and time-dependent expression
}

\author{
Maristela Pereira, Alexandre Melo Bailão, Juliana Alves Parente, Clayton Luiz Borges, \\ Silvia Maria Salem-Izacc, Célia Maria de Almeida Soares/+
}

Laboratório de Biologia Molecular, Instituto de Ciências Biológicas, Universidade Federal de Goiás, 74001-970 Goiânia, GO, Brasil

Paracoccidioides brasiliensis causes infection through inhalation by the host of airborne propagules from the mycelium phase of the fungus. This fungus reaches the lungs, differentiates into the yeast form and is then disseminated to virtually all parts of the body. Here we review the identification of differentially-expressed genes in host-interaction conditions. These genes were identified by analyzing expressed sequence tags (ESTs) from P. brasiliensis cDNA libraries. The P. brasiliensis was recovered from infected mouse liver as well as from fungal yeast cells incubated in human blood and plasma, mimicking fungal dissemination to organs and tissues and sites of infection with inflammation, respectively. In addition, ESTs from a cDNA library of P. brasiliensis mycelium undergoing the transition to yeast were previously analyzed. Together, these studies reveal significant changes in the expression of a number of genes of potential importance in the host-fungus interaction. In addition, the unique and divergent representation of transcripts when the cDNA libraries are compared suggests differential gene expression in response to specific niches in the host. This analysis of gene expression patterns provides details about host-pathogen interactions and peculiarities of sites within the host.

Key words: Paracoccidioides brasiliensis - host-niches - infected tissues

Paracoccidioides brasiliensis is an ascomycete human pathogen that causes paracoccidioidomycosis (PCM), the most prevalent systemic mycosis in Latin America. P. brasiliensis is primarily a respiratory pathogen, infecting the host through the inhalation of airborne propagules from the mycelium phase. In the pulmonary alveolar epithelium, conidia differentiate into the parasitic yeast form. P. brasiliensis is a notably versatile pathogen, able to infect numerous sites within the human body. The infection occurs primarily in the lungs, from where the fungus can disseminate via the bloodstream and/or lymphatic system to all parts of the body, rendering the disseminated form of PCM (Franco 1987). A taxon formerly considered to be a single clonal species, $P$. brasiliensis has recently been reported to be at least three different phylogenetic species (Matute et al. 2006, Carrero et al. 2008). The isolate Pb01, object of the analysis in this review, separates from the three previously-isolated $P$. brasiliensis phylogenetic species. This suggests that it is a new species in the genus Paracoccidioides (Carrero et al. 2008).

In this review, we summarize our studies of infection-related gene expression in P. brasiliensis and highlight the main findings of those studies (Bailão et al. 2006, 2007, Bastos et al. 2007, Costa et al. 2007).

Financial support: CNPq (505658/2004-6; 471808/2006-7)

+ Corresponding author: celia@icb.ufg.br

Received 11 July 2008

Accepted 10 March 2009
Approaches to the study of $P$. brasiliensis gene expression in host-like conditions

One approach used to analyzing $P$. brasiliensis gene expression in host-like conditions involves using cDNA Representational Difference Analysis (cDNA-RDA) Hubank \& Schatz 1994) to identify P. brasiliensis genes induced during the infective process in a mouse model of infection and under conditions that mimicked the hematogenic route of fungal dissemination (Bailão et al. 2006), as well as infection sites with inflammation (Bailão et al. 2007). Subtracted cDNA libraries were made using driver cDNAs from seven-day-old yeast cell cultures and tester cDNAs were synthesized from fungal cell RNAs recovered from the liver of infected animals and from yeast cells after artificial infection with human blood or plasma. The subtracted cDNA libraries and the number of transcripts generated are summarized in Fig. 1. A total of 45, 86 and 99 over-expressed sequences were obtained from the subtracted cDNA libraries of yeast cells recovered from infected liver (Bailão et al. 2006) and from yeast cells incubated with human blood or plasma (Bailão et al. 2006, 2007), respectively, as shown in Fig. 1.

Additionally, Costa et al. (2007) analyzed 4,934 expressed sequence tags (ESTs) from a non-normalized cDNA library derived from $P$. brasiliensis yeast cells recovered from infected mouse liver. Comparative analysis of the unigene sequences at (http://www.lbm.icb. ufg.br/phorestwww/index.php) and the database constructed with fungal sequences of the yeast and mycelium transcriptome (isolate $\mathrm{Pb01}$ ) at (https://dna.biomol. unb.br/Pb/) (Felipe et al. 2005), as well as of all public ESTs available at GenBank (http://www.ncbi.nlm. nih.gov/BLAST/), gave insights into fungal adaptation to this host environment. Cellular differentiation in the 
yeast phase is a fundamental step in the establishment of fungal infection. Therefore, we also analyzed ESTs to assess the expression profile during the mycelium-toyeast transition. Comparative analysis of $P$. brasiliensis ESTs identified in the transition from mycelium to yeast cells (http://192.168.0.5/phorestwww) and the databases cited above allowed the identification of differentiallyexpressed sequences of $P$. brasiliensis during the morphological conversion (Bastos et al. 2007). A summary of the data from non-normalized cDNA libraries is presented in Fig. 2. A total of 1,602 unique sequences were obtained from the non-normalized cDNA libraries of yeast cells recovered from infected mouse liver and 639 were obtained from mycelium in transition to yeast cells (Costa et al. 2007, Bastos et al. 2007). From the 1,602 unique sequences in the cDNA library of yeast cells recovered from infected mice, 649 (40.51\%) represented

\begin{tabular}{|c|c|c|}
\hline & $\begin{array}{c}\text { Subtracted } \\
\text { cDNA libraries }\end{array}$ & \\
\hline Infection in tissue & $\begin{array}{l}\text { Fungal route of } \\
\text { dissemination } \\
\text { (blood) }\end{array}$ & $\begin{array}{l}\text { Sites of infection } \\
\text { with inflamation }\end{array}$ \\
\hline \multicolumn{3}{|l|}{ Mice infection } \\
\hline$\downarrow_{\text {Liver Removel }}$ & P. brasiliensis & $\begin{array}{l}\text { P. brasiliensis } \\
\text { fincubated with }\end{array}$ \\
\hline CFUs & $\begin{array}{l}\text { incubated with } \\
\text { blood }\end{array}$ & $\begin{array}{l}\text { incubated with } \\
\text { plasma }\end{array}$ \\
\hline$\downarrow$ & $\downarrow$ & $\downarrow$ \\
\hline $\begin{array}{c}\text { Subtracted cDNAA } \\
\text { library (RDA) }\end{array}$ & $\begin{array}{c}\text { Subtracted cDNA } \\
\text { library (RDA })\end{array}$ & $\begin{array}{l}\text { Subtracted cDNA } \\
\text { library (RDA ) }\end{array}$ \\
\hline$\downarrow$ & $\downarrow$ & $\downarrow$ \\
\hline \begin{tabular}{|c|}
490 high quality \\
ESTs
\end{tabular} & $\begin{array}{l}417 \text { high quality } \\
\text { ESTs }\end{array}$ & $\begin{array}{l}577 \text { high quality } \\
\text { ESTs }\end{array}$ \\
\hline$\downarrow_{\text {Assembly }}$ & t Asembly & $\downarrow_{\text {Assembly }}$ \\
\hline 45 overexpressed & 86 overexpressed & $99 \begin{array}{c}\text { overexpressed } \\
\text { transcripst }\end{array}$ \\
\hline$\downarrow$ & $\downarrow$ & $\downarrow$ \\
\hline 4 novel & 8 novel & 6 novel \\
\hline transcripts & & \\
\hline
\end{tabular}

Fig. 1: strategies used to identify Paracoccidioides brasiliensis transcripts involved in fungal-host interaction: subtracted cDNA libraries.

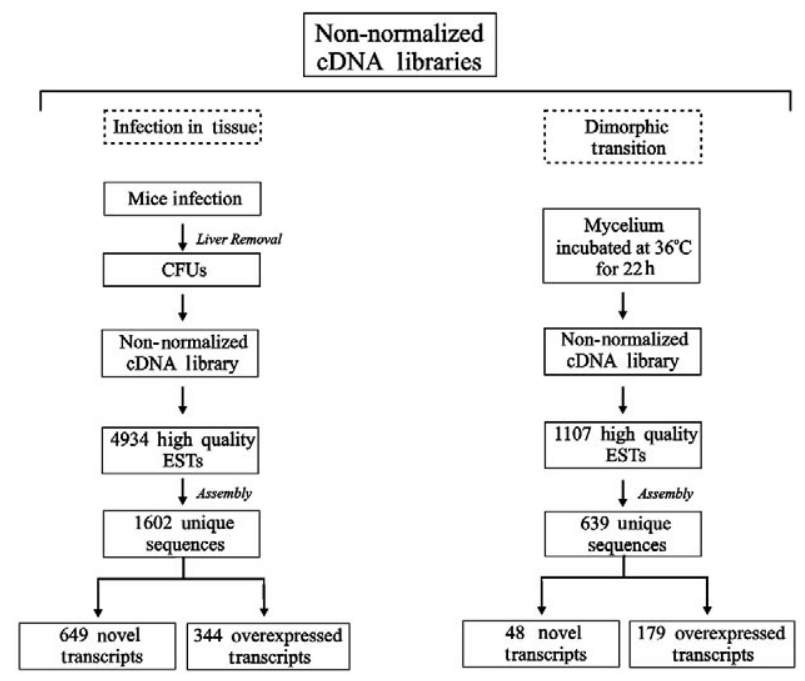

Fig. 2: strategies used to identify Paracoccidioides brasiliensis transcripts involved in fungal-host interaction: non-normalized cDNA libraries. novel transcripts and $344(21.47 \%)$ represented overexpressed genes. Of the 639 unique sequences obtained from sequencing the cDNAs in the non-normalized library of mycelium in transition to yeast cells, there were $48(7.51 \%)$ novel genes and $179(28.01 \%)$ over-expressed transcripts. These data suggest that the $P$. brasiliensis transcriptional profiles during the infective process and differentiation are quite distinct from the transcriptional profile observed in cultured cells.

Using the strategies cited above, we identified a set of candidate genes that $P$. brasiliensis may express in order to adapt to the host. The comparative analysis suggests that $P$. brasiliensis can adapt in both temporal and niche-specific ways to host conditions.

\section{Preferential metabolic pathways that are over-in- duced during liver infection}

Generating precursor metabolites and energy, synthesizing proteins and amino acids, transporting chemicals and responding to environmental stresses are all common responses to host-like conditions. However, certain metabolic processes are presumably more evident in certain fungal treatments, suggesting that the fungal response is more prominent in a defined condition.

By analyzing the most redundant ESTs obtained from cDNAs of yeast cells recovered from the livers of infected mice, it was possible to obtain insight into the features of fungal metabolism during the infective process in tissues (Bailão et al. 2006, Costa et al. 2007). Fig. 3 summarizes the most relevant up-regulated processes, as determined by transcriptional analysis, in yeast cells recovered from liver of infected animals. In liver, $P$. brasiliensis $\mathrm{Pb} 01$ cells induced genes associated with sugar utilization and alcohol fermentation, such as acylphosphatase (E.C.3.6.1.7), quinoprotein alcohol dehydrogenase (E.C.1.1.99.8) and glucokinase (E.C.2.7.1.2). Of special note, and corroborating our data, the predominance of glycolytic metabolism in Candida albicans infecting mouse kidney (Barelle et al. 2006) has been described. The over-expression of carbohydrate metabolism enzymes and overproduction of ethanol suggest that this metabolic pathway could be particularly relevant during liver infection because of the abundant glucose in the host milieu, as described by Costa et al. (2007). The abundance of carbohydrate and energy in this infection condition could also pertain to the over-expression of transcripts encoding lipid-biosynthesis enzymes.

The glyoxylate cycle, required for growth in carbon sources of less than three carbon atoms (such as ethanol or acetate) is an important anaplerotic device. Our in silico subtractive analysis indicated that the cycle is up-regulated in yeast cells during liver infection, relative to in vitro cultured yeast cells, as demonstrated by the over-expression of the regulatory enzyme isocitrate lyase (Costa et al. 2007). This gene is up-regulated in C. albicans during growth inside macrophages (Lorenz \& Fink 2001).

\section{Preferential metabolic pathways that are over-induced during artificial infection in human blood and plasma}

The most prominent adaptations performed by $P$. brasiliensis during treatment with human blood and 


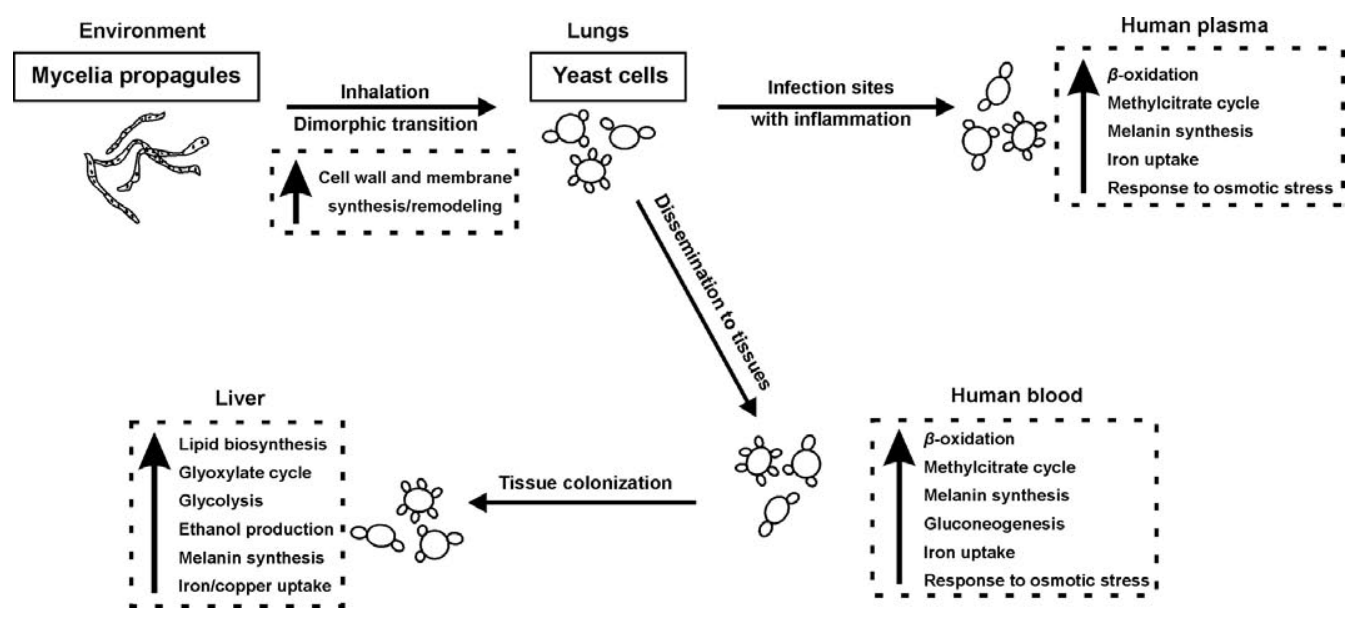

Fig. 3: the most prominent metabolic processes upregulated in conditions mimicking host conditions. Descriptions are summarized from the detailed data presented in Bailão et al. (2006, 2007), Bastos et al. (2007) and Costa et al. (2007).

plasma are summarized in Fig. 3. The degradation of fatty acids through $\beta$-oxidation could be inferred, since all gene homologues encoding enzymes in this pathway were up-regulated following exposure to blood and plasma (Bailão et al. 2006, 2007). Interestingly, induction of methylcitrate genes such as the transcript encoding to 2-methylcitrate dehydratase (E.C.4.2.1.79) (which converts methylcitrate to methylisocitrate) was observed. The assembled data indicate the predominance of lipolytic metabolism in fungal cells exposed to human blood and plasma. Gluconeogenesis is probably up-regulated in yeast cells incubated in human blood, as inferred by the over-expression of the gene encoding phosphoenolpyruvate carboxykinase (E.C.4.1.1.32).

\section{The most prominent metabolic pathways induced during the dimorphic transition}

As inferred from transcriptional data, the most prominent events during the initial stages of the fungal transition from mycelium to yeast cells would seem to be the remodelling of the membrane and cell wall (Bastos et al. 2007). The dimorphic transition occurs simultaneously with changes in the composition of the fungal cell wall (San-Blas et al. 2002). As described by Bastos et al. (2007), transcription of 34-cell wall/membranerelated genes was induced upon temperature shift. The main polysaccharide of the yeast cell wall is alpha-1.3 glucan, while the mycelium contains predominantly beta-glucan (Kanetsuna et al. 1969). Several genes related to the synthesis of carbohydrate cell wall components (such as alpha-1.3 glucan) were induced in the transition library relative to the mycelium transcriptome database (http://www.dna.biomol.unb.br/Pb), putatively enabling an increase in the synthesis of alpha-1,3 glucan for the differentiation process and establishment of yeast cells. Remodelling of the cell wall during the fungal transition is reflected in the transcriptional analysis by the over-expression, for example, of the genes encoding chitinase 1 (CTS1) and 3 (CTS3), as well as UDP-N-acetyl glucosamine transporter, which putatively provides $\mathrm{N}-$ acetyl glucosamine for the synthesis of chitin. Thus, microarray hybridization analysis identified the up-regulation of a series of genes encoding enzymes related to the changes in fungal surface in $P$. brasiliensis, isolate $\mathrm{Pb} 18$ (Nunes et al. 2005).

There were also increases in the expression of transcripts encoding enzymes associated with the synthesis of phospholipids and polyunsaturated fatty acids. The data support the concept that the developmental program of $P$. brasiliensis is characterized by significant remodelling of the cell surface, suggesting the relevance of this process as a contributor to fungal dimorphism.

\section{Over-expressed metabolic pathways putatively re- lated to fungal virulence}

Some metabolic processes are putatively associated with fungal virulence (Fig. 3). The over-expression of genes related to iron uptake has been demonstrated in the cDNA libraries of yeast cells recovered from liver of infected mice (Bailão et al. 2006, Costa et al. 2007) and of yeast cells incubated with human blood or plasma (Bailão et al. 2006, 2007), suggesting that $P$. brasiliensis experiences iron deprivation under those conditions. Iron, which is a metal prominently involved in the transfer of electrons during metabolism, is of great importance to the virulence of mammalian pathogens. It has been demonstrated, for instance, that iron overload exacerbates miningioencephalities in a mouse model of cerebral infection by Cryptococcus neoformans (Barluzzi et al. 2002). The gene CIRI (Cryptococcus iron regulator), which encodes the major regulator of the response to iron in $C$. neoformans, influences the transcription of iron transport and homeostasis functions and controls the expression of virulence functions such as capsule, melanin and growth at host temperature (Jung et al. 2008).

Interconnections between copper and iron homeostasis are well established in fungi. The high-affinity system of iron uptake, which envolves a member of the copper 
oxidase family, is present in fungi (Stearman et al. 1996) and requires efficient copper uptake, since maturation of the copper protein in the secretory apparatus requires the acquisition of copper and the absence of genes related to this activity results in a decrease in high affinity iron transport (Dancis et al. 1994). Transcripts for iron and copper transporters were elevated in C. neoformans during early murine pulmonary infection, indicating a nutrient-limited host environment (Hu et al. 2008). The transport of copper seems to be exacerbated in P. brasiliensis yeast cells recovered from liver of infected mice. The results suggest that there is an iron-regulated, high-affinity iron uptake system in P. brasiliensis and that those genes are regulated in response to iron starvation during host infection. In addition, a system of low-affinity iron uptake seems to occur in P. brasiliensis, as inferred from the expression of ferric reductase and low-affinity iron transport permease (Bailão et al. 2007). In C. albicans there are two genes encoding iron permease and the high affinity transporter is required for virulence in a mouse model of infection (Ramanan \& Wang 2000).

Transcripts encoding enzymes for the synthesis of melanin were induced in the following experimental conditions. The transcript encoding aromatic L-amino acid decarboxylase (E.C.4.1.1.28) was over-expressed in yeast cells recovered from murine infection (Bailão et al. 2006) and in yeast cells incubated with human blood and plasma (Bailão et al. 2006, 2007). This reinforces the relevance of DOPA-melanin in infection, as evidenced in previous studies (Silva et al. 2006). Of special note is the detection of the transcript encoding polyketide synthase (E.C.1.3.1.10) during liver infection. This suggests that $P$. brasiliensis could also synthesize dihydroxynaphtalene (DHN)-melanin, as described in some fungi (Paolo Jr et al. 2006).

\section{Time-dependent gene expression in $P$. brasiliensis in host-like conditions}

Fig. 4 summarizes differential expression data over the time course of fungal incubation with human blood by
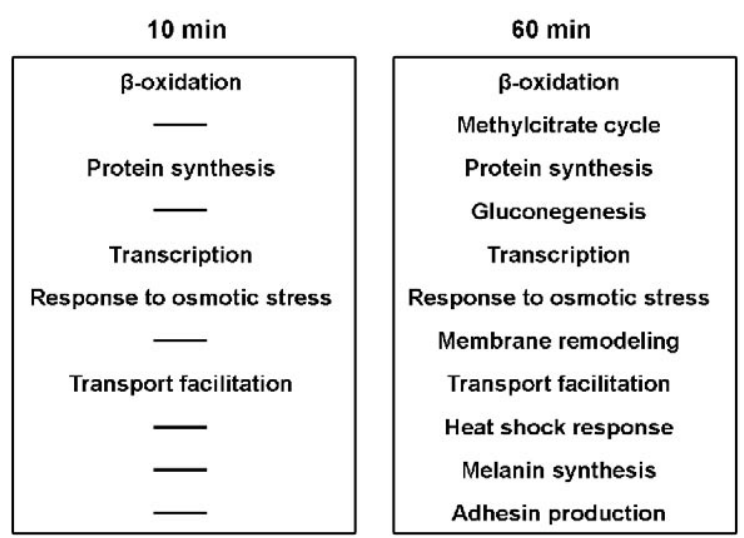

Fig. 4: summary of some cellular processes that are associated with genes upregulated at different time points of yeast cells treatment with human blood. Descriptions are summarized from the detailed data presented in Bailão et al. (2006). listing processes that are important at each stage. Genes encoding $\beta$-oxidation, protein synthesis, transcription, response to osmotic stress and transport facilitation were induced at both 10 and $60 \mathrm{~min}$ time points. As the incubation continued, the expression of genes encoding proteins involved in the methylcitrate cycle, gluconeogenesis, membrane remodelling, heat shock response, melanin synthesis and adhesion were up-regulated. Of 86 genes induced in yeast cells upon exposure to human blood, 85 were differentially expressed as a function of time (Bailão et al. 2006). Incubation with human plasma stimulated expression of 94 genes out of a total of 99 differentially induced as a function of time (Bailão et al. 2007). It is plausible that this time-dependent gene expression could reflect the progressive adaptation of fungus to the new environment. The differential expression of genes as a function of time has been described in C. albicans during planktonic culture growth (Yeater et al. 2008).

\section{Niche-regulation of genes related to cell rescue and virulence}

Some transcripts seem to be specifically induced in particular host conditions and are good candidates for host niche-specific responses (Fig. 5). We observed an over-expression of iron uptake systems in the cDNA libraries obtained from $\mathrm{Pb} 01$ yeast cells recovered from infected liver and from human blood and plasma. However, a striking feature of this over-expression is that, in the last two conditions, the process seems to involve a ferric reductase that may belong to a low-affinity elemental iron transport system, as described in other fungi (Georgatsou \& Alexandraki 1994), possibly reflecting differential iron availability in the analyzed conditions.

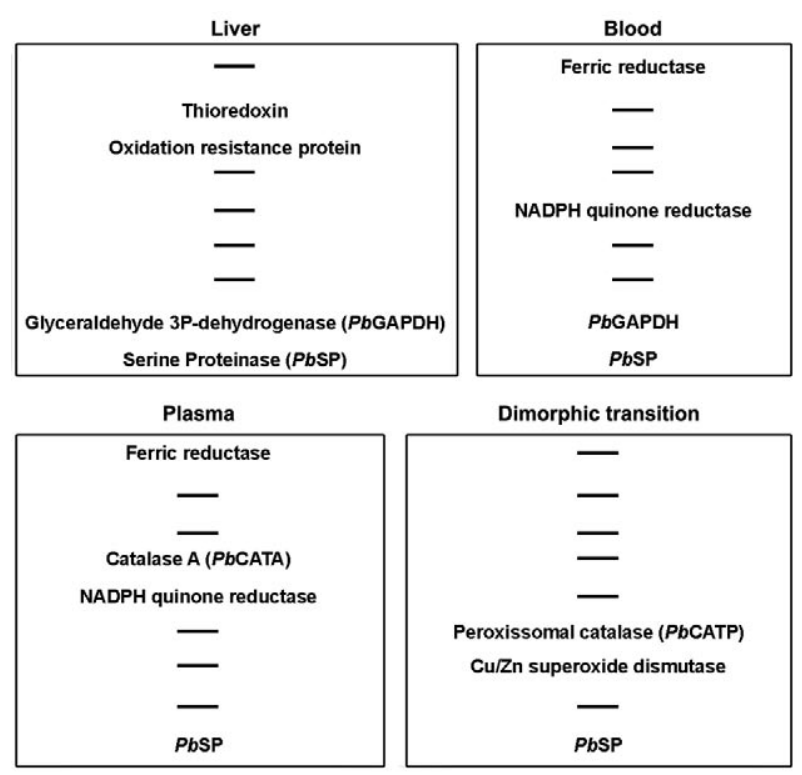

Fig. 5: summary of some candidate genes related to cell rescue and virulence upregulated in different host-like conditions. Descriptions are summarized from the detailed data presented in Bailão et al. (2006, 2007), Bastos et al. (2007) and Costa et al. (2007). 
Although the response to oxidative stress seems to be a general feature in all the experimental conditions reviewed here, the specific molecules that are upregulated seem to vary in the analyzed conditions. As examples, in yeast cells recovered from liver infection we detected induced transcripts encoding homologues of thioredoxin, an oxidation resistance protein; in yeast cells in the presence of plasma, the expression of the homologue to catalase A was detected; and NADPH-quinone reductase was detected during yeast cell incubation with blood and plasma. During the 22-h transition from mycelium to yeast the fungus preferentially expressed transcripts encoding catalase $\mathrm{P}$ and $\mathrm{Cu} / \mathrm{Zn}$ superoxide dismutase. It has been suggested that superoxide dismutases, which promote the rapid degradation of superoxide into hydrogen peroxide and $\mathrm{O}_{2}$, augment adaptation of $C$. neoformans to human host body temperature (Giles et al. 2005). These observations suggest a nichespecific activation of the oxidative stress response by $P$. brasiliensis, as described for $C$. albicans (Enjalbert et al. 2007). Corroborating these observations, we recently demonstrated that $P$. brasiliensis responds to oxidative, osmotic and heat stresses by differentially up-regulating the catalases A, C and P (Chagas et al. 2008).

Virulence attributes such as adhesins and secreted hydrolases are regulated during infection. Glyceraldehyde-3-phosphate-dehydrogenase (GAPDH) is induced in $\mathrm{Pb} 01$ obtained from infected mouse liver and in yeast cells incubated in blood (Bailão et al. 2006). The GAPDH of $P$. brasiliensis reacts with the serum of PCM patients (Fonseca et al. 2001, Barbosa et al. 2004) and is located at the fungal cell wall. This protein is a $P$. brasiliensis adhesin that binds components of the extracellular matrix and is able to mediate the adherence and internalization of $P$. brasiliensis to in vitro-cultured cells, suggesting its involvement in the pathogenesis of the fungus (Barbosa et al. 2006).

Interestingly, some genes related to cell rescue and virulence were over-induced in all conditions analyzed (Bailão et al. 2006, 2007, Bastos et al. 2007, Costa et al. 2007). One such example is the transcript encoding a serine proteinase (E.C.3.4.21.4). Several lines of evidence suggest that serine proteinases are required for the successful invasion of host cells by pathogens. An extracellular SH-dependent serine proteinase has been characterized in the yeast phase of $P$. brasiliensis. It cleaves the main components of the basal membrane in vitro and is thus potentially relevant to fungal dissemination (Puccia et al. 1999). Serine proteinases could play an important role in the cleavage of host proteins either during the invasion of a host cell or during dissemination through organs.

\section{Perspectives}

The data obtained from these transcriptional analyses have broadened our knowledge of the mechanisms used by this fungus to differentiate and adapt to the host environment. In a complementary approach, we will analyze the proteome of $P$. brasiliensis, as well as the protein-protein interactions of molecules potentially associated with the process of infection.
For an initial characterization of $P$. brasiliensis protein-protein interactions, we conducted yeast two hybrid $(\mathrm{Y} 2 \mathrm{H})$ assays using selected proteins previously characterized in our laboratory (manuscript in preparation). These proteins are probably involved in the fungus' infective process, as suggested in previous studies (Bailão et al. 2006, 2007, Costa et al. 2007) and they were classified into different functional categories such as cell adhesion, micronutrient uptake, transport, protein degradation and cell wall metabolism. The selected proteins were used as bait against a cDNA library constructed with mRNAs obtained from $P$. brasiliensis yeast cells recovered from liver of infected mice. Several relevant interactions were detected, reinforcing the role of the analyzed proteins in their functional categories and also pointing to their function in the infective process of $P$. brasiliensis.

In a second and more enlightening approach, highthroughput $\mathrm{Y} 2 \mathrm{H}$ studies are being performed. These studies of $P$. brasiliensis' interactome should help to decipher the enzymatic complexes, signalling and metabolic pathways, and binding event cascades that contribute to $P$. brasiliensis' adaptation and survival during the infective process.

In summary, using a comparative analysis approach, we identified sequences potentially relevant to the ability of $P$. brasilensis to cause infection and to adapt to host niches. Taken together, the results reveal specific metabolic adaptations of this fungus during infection. Gene cataloguing and profiling of $P$. brasiliensis yeast cells recovered from infected animal tissues and other conditions mimicking sites of infection is an essential prerequisite for elucidating mechanisms of fungal virulence and pathogenesis. These results provide important information for future research on the infective process of this fungal pathogen.

\section{REFERENCES}

Bailão AM, Schrank A, Borges CL, Dutra V, Molinari-Madlum EEWI, Felipe MSS, Mendes-Giannini MJS, Martins WS, Pereira M, Soares CMA 2006. Differential gene expression by Paracoccidioides brasiliensis in host interaction conditions: Representational difference analysis identifies candidate genes associated with fungal pathogenesis. Microbes Infect 8: 2686-2697.

Bailão AM, Shrank A, Borges CL, Parente JA, Dutra V, Felipe MSS, Fiúza RB, Pereira M, Soares CMA 2007. The transcriptional profile of Paracoccidioides brasiliensis yeast cells is influenced by human plasma. FEMS Immunol Med Microbiol 51: 43-57.

Barbosa MS, Bao NS, Andreotti PF, Felipe MSS, Feitosa LS, MendesGiannini MJS, Soares CMA 2006. Glyceraldehyde 3-phosphate dehydrogenase of Paracoccidioides brasiliensis is a cell surface protein, involved in fungal adhesion to extracellular matrix proteins and interaction with cells. Infect Immun 74: 382-389.

Barbosa MS, Cunha Passos DA, Felipe MSS, Jesuíno RSA, Pereira M, Soares CMA 2004. The glyceraldehyde-3-phosphate dehydrogenase homologue is differentially regulated in phases of Paracoccidioides brasiliensis: molecular and phylogenetic analysis. Fungal Genet Biol 41: 667-675.

Barelle CJ, Priest CL, MacCallum DM, Gow NAR, Odds FC, Brown AJP 2006. Niche-specific regulation of central metabolic pathways in a fungal pathogen. Cell Microbiol 8: 961-971.

Barluzzi R, Saleppico S, Nocentini A, Boelart JR, Neglia R, Bistoni F, Blasi E 2002. Iron overload exacerbates experimental menin- 
goencephalitis by Cryptococcus neoformans. J Neuroimmunol 132: 140-146.

Bastos KP, Bailão AM, Borges CL, Faria FP, Felipe MSS, Silva MG, Martins WS, Fiúza RB, Pereira M, Soares CMA 2007. The transcriptome analysis of early morphogenesis in Paracoccidioides brasiliensis mycelium reveals novel and induced genes potentially associated to the dimorphic process. BMC Microbiol 7: 29.

Carrero LL, Niño-Vega G, Teixeira MM, Carvalho MJA, Soares CMA, Pereira M, Jesuino RSA, McEwen JG, Mendoza L, Taylor JW, Felipe MSS, San-Blas G 2008. New Paracoccidioides brasiliensis isolate reveals unexpected genomic variability in this human pathogen. Fungal Genet Biol 45: 605-612.

Chagas RF, Bailão AM, Pereira M, Winters SM, Smullian AG, Deepe Jr GS, Soares CMA 2008. The catalases of Paracoccidioides brasiliensis are differentially regulated: protein activity and transcript analysis. Fungal Genet Biol 45: 1470-1478.

Costa M, Borges CL, Bailão AM, Meirelles GV, Mendonça YA, Dantas SFIM, Faria FP, Felipe MSS, Molinari-Madlum EEWI, Mendes-Giannini MJS, Fiúza RB, Martins WS, Pereira M, Soares CMA 2007. Transcriptome profiling of Paracoccidioides brasiliensis yeast-phase cells recovered from infected mice brings new insights into fungal response upon host interaction. Microbiology 153: 4194-4207.

Dancis A, Haile D, Yuan DS, Klausner RD 1994. The Saccharomyces cerevisiae copper transport protein (Ctrlp). Biochemical characterization, regulation by copper and physiologic role in copper uptake. J Biol Chem 269: 25660-25667.

Enjalbert B, MacCallum DM, Odds FC, Brown AJP 2007. Niche-specific activation of the oxidative stress response by the pathogenic fungus Candida albicans. Infect Immun 75: 2143-2151.

Felipe MSS, Andrade RV, Arraes FB, Nicola AM, Maranhão AQ, Torres FA, Silva-Pereira I, Poças-Fonseca MJ, Campos EG, Moraes LM, Andrade PA, Tavares AH, Silva SS, Kyaw CM, Souza DP, Pereira M, Jesuíno RS, Andrade EV, Parente JA, Oliveira GS, Barbosa MS, Martins NF, Fachin AL, Cardoso RS, Passos GA, Almeida NF, Walter ME, Soares CMA, Carvalho MJ, Brígido MM, Pbgenome Network 2005. Transcriptional profiles of the human pathogenic fungus Paracoccidioides brasiliensis in mycelium and yeast cells. J Biol Chem 280: 24706-24714.

Fonseca CA, Jesuino RSA, Felipe MSS, Cunha DA, Brito WA, Soares CMA 2001. Two-dimensional electrophoresis and characterization of antigens from Paracoccidioides brasiliensis. Microbes Infect 3: 535-342.

Franco M 1987. Host-parasite relationship in paracoccidioidomycosis. J Clin Microbiol 25: 5-18.

Georgatsou E, Alexandraki D 1994. Two distinctly regulated genes are required for ferric reduction, the first step of iron uptake in Saccharomyces cerevisiae. Mol Cell Biol 14: 3065-3073.

Giles S, Batinick-Haberle I, Perfect J, Cox G 2005. Cryptococcus neoformans mitochondrial superoxide dismutase and essential link between antioxidant function and high-temperature growth. Eukaryot Cell 4: 46-54.
Hu G, Cheng PY, Sham A, Perfect JR, Kronstad JW 2008. Metabolic adaptation in Cryptococcus neoformans during early murine pulmonary infection. Mol Microbiol 69: 1456-1475.

Hubank M, Schatz DG 1994. Identifying differences in mRNA expression by representational difference analysis of cDNA. Nucleic Acids Res 22: 5640-5648.

Jung WH, Sham A, Lian T, Singh A, Kosman DJ, Kronstad JW 2008. Iron source preference and regulation of iron uptake in Cryptococcus neoformans. PLoS Pathog 4: e45.

Kanetsuna F, Carbonell LM, Moreno RE, Rodriguez J 1969. Cell wall composition of the yeast and mycelial forms of Paracoccidioides brasiliensis. J Bacteriol 97: 1036-1041.

Lorenz MC, Fink GR 2001. The glyoxylate cycle is required for fungal virulence. Nature 412: 83-86.

Matute DR, McEwen JG, Puccia R, Montes BA, San-Blas G, Bagagli E, Rauscher JT, Restrepo A, Morais F, Niño-Vega G, Taylor JW 2006. Cryptic speciation and recombination in the fungus Paracoccidioides brasiliensis as revealed by gene genealogies. Mol Biol Evol 23: 65-73.

Nunes LR, Oliveira RC, Leite DB, Silva VS, Marques ER, Ferreira MES, Ribeiro DCD, Bernardes LAS, Goldman MHS, Puccia R, Travassos LR, Batista WL, Nóbrega MP, Nóbrega FG, Yang DY, Pereira CAB, Goldman GH 2005. Transcriptome analysis of Paracoccidioides brasiliensis cells undergoing mycelium-toyeast transition. Eukaryot Cell 4: 2115-2128.

Paolo Jr WF, Dadachova E, Mandal P, Casadevall A, Szsaniszlo PJ, Nosanchuk JD 2006. Effects of disrupting the polyketide synthase gene WdPKS1 in Wangiella [Exophiala] dermatitidis on melanin production and resistance to killing by antifungal compounds, enzymatic degradation, and extremes in temperature. BMC Microbiol 19: 55.

Puccia R, Juliano MA, Juliano L, Travassos LR, Carmona AK 1999. Detection of the basement membrane-degrading proteolytic activity of Paracoccidioides brasiliensis after SDS-PAGE using agarose overlays containing Abz-MKALTLQ-EDDnp. Braz $J$ Med Biol Res 32: 645-649.

Ramanan N, Wang Y 2000. A high affinity iron permease essential for Candida albicans virulence. Science 288: 1062-1069.

San-Blas G, Niño-Vega G, Iturriaga T 2002. Paracoccidioides brasiliensis and paracoccidioidomycosis: molecular approaches to morphogenesis, diagnosis, epidemiology, taxonomy and genetics. Med Mycol 40: 225-242.

Silva MB, Marques AF, Nosanchuck JD, Casadevall A, Travassos LR, Taborda CP 2006. Melanin in the dimorphic fungal pathogen Paracoccidioides brasiliensis: effects on phagocytosis, intracellular resistance and drug susceptibility. Microbes Infect 8: 197-205.

Stearman R, Yuan DS, Yamaguchi-Iwai Y, Klausner RD, Dancis A 1996. A permease-oxidase complex involved in high-affinity iron uptake in yeast. Science 271: 1552-1557.

Yeater KM, Chandra J, Cheng G, Mukherjee PK, Zhao X, RodriguesZas SL, Kwast KE, Ghannoum MA, Hoyer LL 2008. Temporal analysis of Candida albicans gene expression during biofilm development. Microbiology 153: 2373-2385. 